\begin{tabular}{lll}
$\begin{array}{l}\text { Abstract 57 Table 1 } \\
\text { cases }\end{array}$ & Summary of both LVOT pseudoaneurysm \\
\hline Clinical characteristic & Patient 1 & Patient 2 \\
\hline $\begin{array}{l}\text { Age (years) } \\
\text { Sex }\end{array}$ & 69 & 76 \\
Index aortic valve pathology & Female & Female \\
& $\begin{array}{l}\text { Mixed bicuspid aortic } \\
\text { valve disease }\end{array}$ & $\begin{array}{l}\text { Severe trileaflet aortic } \\
\text { stenosis }\end{array}$ \\
SAVR details & $25 \mathrm{~mm}$ St Jude Trifecta & $19 \mathrm{~mm}$ St Jude Trifecta \\
SAVR to diagnosis of LVOT & 55 & 60 \\
pseudo-aneurysm (days) & & \\
Symptoms due to LVOT & None \\
pseudo-aneurysm & & Chest pain and \\
Pseudo-aneurysm dimensions (mm) & $5 \times 23 \times 25 \mathrm{~mm}$ & dyspnoea \\
Vascular access & Trans-femoral & Trans-femoral \\
& (catheter) & (catheter) \\
Device used & AVP2 $10 \mathrm{~mm}$ & AVP2 $10 \mathrm{~mm}$ \\
\hline
\end{tabular}

evolving percutaneous structural heart interventions it is highly important that we possess a thorough understanding of the many percutaneous devices and treatment options available for post-surgical complications.

\section{IMPLEMENTING THE JACC CONSENSUS STATEMENT PATHWAYS FOR MANAGEMENT OF CONDUCTION DISTURBANCES POST -TAVI - THE EFFECT ON INPATIENT LENGTH OF STAY}

P Wheen. L Byrne, M Alenezi, S O'Connor. St. James's Hospital, Dublin, Ireland

\subsection{6/heartjnl-2020-ICS.58}

Background Transcatheter Aortic Valve Implantations (TAVIs) are associated with a need for Permanent Pacemaker (PPM) insertion with a frequency between $2.3 \%-36.1 \%$ at 30 days post procedure. Although the indication for PPM is often apparent at the time of TAVI, many patients develop indications for PPM in a delayed manner following TAVI, and can have fatal outcomes when they present with delayed high degree AV block, leading to uncertainty around duration of monitoring following TAVI. In 2019, the JACC Consensus Statement was published to help guide physicians in this regard.

Methods We reviewed patients who underwent TAVI in our centre who had complete follow up data between 2018-2019. We categorised them according to the groups suggested in the consensus document. We assessed their subsequent PPM implantation rate at 30 days, and reviewed their length of inpatient admission.

Results 56 patients (37 males) without prior PPM underwent TAVI in our centre in the study period. There were 30 patients in group 1 (no ECG changes without pre-existing right bundle branch block (RBBB)), all alive at 30 days, of whom $2(6.7 \%)$ underwent a PPM within 30 days of TAVI. Their median length of stay was 3 days (2-6). There were 5 patients in group 2 (no new ECG changes, with pre existing $\mathrm{RBBB})$; of these none required a PPM at 30 days, with a median LOS of 8 days (4.5-8). 5 patients in group 3 (New ECG changes in patients with pre-existing conduction disease) had a median length of stay of 5 days (3-17), 4 of whom required PPM by 30 days. 7 patients in group 4 (new left bundle branch block (LBBB)) median length of stay was 5 days (3-7), of whom $1(14.2 \%)$ required a PPM. 2 patients in group 5, of whom both required PPM, and median length of stay was 4 days (3.5-4.5). There was only 1 patient (in group 2) who was discharged prior to the recommended length of monitoring as per the Consensus statement. The risk categorisation is effective for determining high risk patients for monitoring.

Conclusion Implementation of the JACC consensus statement does not significantly lengthen the duration of inpatient stay in our institution. However, it effectively stratifies risk of PPM in patients following TAVI, and could be helpful in determining length of rhythm monitoring.

\section{DAPT: ARE WE GETTING THE BALANCE RIGHT?}

${ }^{1}$ B Traynor, ${ }^{1}$ A Dromey, ${ }^{1} \mathrm{MJ}$ Daly, ${ }^{1} \mathrm{~A}$ Mansur, ${ }^{1} \mathrm{~K}$ Hasan, ${ }^{2} \mathrm{E}$ Keelan, ${ }^{2} \mathrm{~J}$ Galvin, ${ }^{1} \mathrm{~J}$ O'Neill. ${ }^{1}$ Connolly Hospital Blanchardstown, Dublin, Ireland; ${ }^{2}$ Heart and Vascular Cardiology Department, Mater Hospital Dublin Ireland

\subsection{6/heartjnl-2020-ICS.59}

Introduction Dual Anti-Platelet Therapy (DAPT) is indicated in Acute Coronary Syndrome (ACS) management and following elective Percutaneous Coronary Intervention (PCI). Optimal duration to balance the risks of bleeding and ischaemic events, including stent thrombosis, is an evolving debate with the advent of newer-generation Drug-Eluting Stents (DES) and evidence from the most recent randomised controlled trials. It should also be tailored to indication and individual patient risk profile. Guidelines concerning DAPT were published by the European Society of Cardiology in 2017 with risk stratification tools to estimate individual bleeding and ischaemic risk to guide and inform these decisions. Our aim was to establish current practice of DAPT prescribing in our department and investigate for adherence to guidelines.

Methods Data were collated for 54 prospectively and randomly chosen patients attending general cardiology clinic between February and April 2020 who had commenced DAPT between 12 and 60 months previously following ACS or elective PCI. Patients given DAPT for other indications, or prior to elective angiography where DAPT did not continue thereafter, were not included. Four patients were excluded as insufficient data were available. 'PRECISE DAPT Score' at initiation and 'DAPT score' 12 months post initiation were retrospectively calculated. Demographic and clinical data were sourced from chart review and electronic laboratory and radiology systems. Data were expressed as mean \pm SD and $\%$.

Results Fifty patients were included of whom 39 were male and median age was 60.5 years (range 35-90). Of this cohort, $64 \%$ were current or ex-smokers, $14 \%$ had diabetes, $58 \%$ had hypertension, $56 \%$ had documented family history of ischaemic heart disease and $46 \%$ had hyperlipidaemia. PRECISE DAPT score calculated bleed risk was very low in $24 \%$, low in $42 \%$, moderate in $20 \%$ and high in $14 \%$. Mean risk of TIMI major or minor grade bleeding during 12 months DAPT was $1.21 \%$ using this score. DAPT score at 12 months following initiation was low in $44 \%$ and high in $56 \%$.

DAPT Indication was ACS in 62\%, elective PCI in $36 \%$ and 1 patient underwent 12 months DAPT inappropriately after elective angiography without PCI. Overall, 86\% received DESs, 4\% drug-eluting balloons and 10\% medical therapy. Almost half $(48 \%)$ did not receive the DAPT duration specified by the cardiologist or based on ESC guidelines where 